\title{
A!
}

This is an electronic reprint of the original article.

This reprint may differ from the original in pagination and typographic detail.

Haq, Faizan UI; Englund, Mikko; Stadius, Kari; Kosunen, Marko; Ryynanen, Jussi; Koli, Kimmo; Ostman, Kim B.

A wideband blocker-resilient direct $\Delta \Sigma$ receiver with selective input-impedance matching

Published in:

IEEE International Symposium on Circuits and Systems

DOI:

10.1109/ISCAS.2017.8050249

Published: 25/09/2017

Document Version

Peer reviewed version

Please cite the original version:

Haq, F. U., Englund, M., Stadius, K., Kosunen, M., Ryynanen, J., Koli, K., \& Ostman, K. B. (2017). A wideband blocker-resilient direct receiver with selective input-impedance matching. In IEEE International Symposium on Circuits and Systems: From Dreams to Innovation, ISCAS 2017 - Conference Proceedings [8050249] (IEEE International Symposium on Circuits and Systems proceedings). IEEE.

https://doi.org/10.1109/ISCAS.2017.8050249

This material is protected by copyright and other intellectual property rights, and duplication or sale of all or part of any of the repository collections is not permitted, except that material may be duplicated by you for your research use or educational purposes in electronic or print form. You must obtain permission for any other use. Electronic or print copies may not be offered, whether for sale or otherwise to anyone who is not an authorised user. 


\section{A Wideband Blocker-Resilient Direct $\Delta \Sigma$ Receiver With Selective Input-Impedance Matching}

\author{
Faizan Ul Haq, Mikko Englund, Kari Stadius \\ Marko Kosunen, Jussi Ryynänen \\ Dept. of Micro and Nano Sciences \\ Aalto university, Espoo Finland \\ Email: faizan.ulhaq@aalto.fi
}

Kimmo Koli

Huawei Technologies Oy Co. Ltd

Finland

Email: kimmo.koli@huawei.com Email: kim.ostman@nordicsemi.no

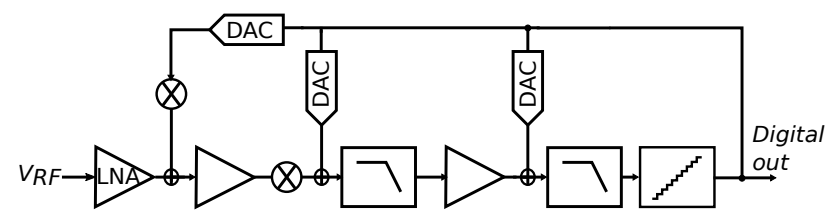
included in the $\Delta \Sigma$ modulator (DSM) loop-filter.

\begin{abstract}
This paper presents a wideband blocker-tolerant Direct $\Delta \Sigma$ receiver (DDSR). Blockers are attenuated through selective input impedance matching and optimized gain design. The created impedance profile provides low receiver input impedance at blocker frequencies, while at desired frequencies, the impedance is boosted to matched condition through an upconverted positive feedback from the DDSR output. Receiver is evaluated in a $28 \mathrm{~nm}$ fully-depleted silicon-on-insulator CMOS process with total power consumption of $25 \mathrm{~mW}$ at $1 \mathrm{~V}$ supply voltage. The receiver is designed for configurable operation from $0.7-2.7 \mathrm{GHz}$, a baseband bandwidth of $10 \mathrm{MHz}$, demonstrates a maximum noise figure of $6.2 \mathrm{~dB}$, and achieves a peak SNDR of $53 \mathrm{~dB}$ with an out-of-band $1 \mathrm{~dB}$ input compression point of $-11.5 \mathrm{dBm}$ at $100 \mathrm{MHz}$ offset.
\end{abstract}

\section{INTRODUCTION}

Digital intensive wideband receivers, for emerging radio access standards such as 5G and LTE, are in growing demand due to their ease of configurability. One such digital intensive architecture is the direct $\Delta \Sigma$ receiver (DDSR) as illustrated in Figure 1 [1]. The architecture differs from the conventional direct conversion receiver by embedding RF front-end blocks as a part of delta-sigma-modulator (DSM) loop-filter. In this way, signal discretization already begins at RF and the DSM loop-filter now performs both functions of channel select filtering (CSF) and quantization noise shaping.

Inherently, such wideband DDSRs are exposed to highpower out-of-band (OB) blockers. If not attenuated, these blockers can completely saturate the receiver (RX) and make the RX operation non-linear. Techniques such as applying $\mathrm{N}$ path filtering at the LNA output and low-noise transconductance amplifier (LNTA)/mixer first arrangements have been reported earlier for OB blocker attenuation [2], [3]. However, as will be explained later, all these techniques have certain limitations when it comes to blocker attenuation.

Compared to previously introduced techniques, the blockerresilient DDSR proposed in this paper adds additional bandpass filtering at the DDSR input through selective input impedance matching. Additionally, an approach for optimized receiver gain design is followed which results in an improved near-band compression point.

The paper is organized as follows. Section II describes the limitations of blocker rejection with basic N-path filtering and how the blocker rejection can be improved. Section III
Fig. 1. Generic block diagram of the direct $\Delta \Sigma$ receiver. RF stages are

details theoretical aspect of proposed DDSR while section IV covers its transistor level implementation details. The paper finishes with performance evaluation results in section $\mathrm{V}$ and conclusion in section VI.

\section{BLOCKER REJECTION FOR A DDSR}

Typically, a DDSR implements basic N-path filtering techniques for OB blocker rejection at the LNA output [1], [4]. However, there are two limitations with this approach. First, the gain/loss of far-away blockers is limited by the N-path mixer switch resistance $\left(\mathrm{R}_{\mathrm{ON}}\right)$ and the LNA transconductance $\left(\mathrm{gm}_{\mathrm{LNA}}\right)$ which should both be minimized. These parameters cannot be reduced indefinitely due to limitations on LO drive power consumption and LNA noise contribution. Second, filtering is implemented only at the output of the LNA, neglecting the filtering requirement at the LNA input. Without input filtering and provided the low blocker gain at the LNA output, LNA transconductor can enter non-linear operation if the blocker reaches the input swing limitations of the transconductor. Therefore, an optimum design should ideally filter the OB blockers already at the LNA input. Input transconductor non-linearity can also be overcome by completely eliminating it in mixer first arrangements. However, in DDSRs, mixer first arrangments pose quantization noise image emission to antenna [1]. Therefore, mixer first topology is problematic for our application.

Another important aspect for optimized OB blocker rejection is to only apply the minimum possible gain required in the RX chain. For basic N-path filters, lower gain will result in lower near-band blocker gain and consequently improve the near-band compression point. However, generally gain cannot 


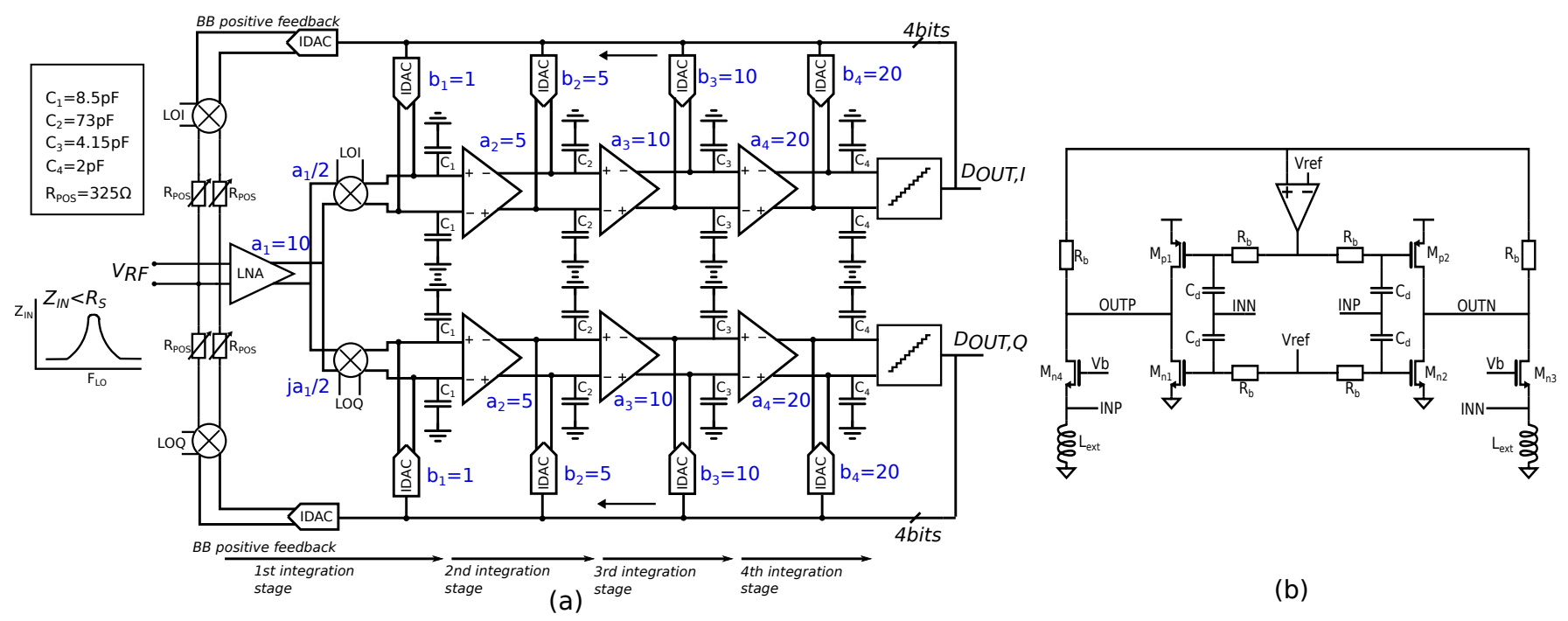

Fig. 2. (a) Proposed wide-band DDSR architecture. Blockers are attenuated through low LNA input impedance ( $\left.\mathrm{Z}_{\mathrm{IN}}\right)$ while positive feedback from BB increases the $Z_{\mathrm{IN}}$ to matched condition for desired frequency (b) Common-Gate Common-Source (CG-CS) LNA schematic.

be reduced indefinitely as it's needed for two reasons. First, it suppresses noise contributions from the later stages of the receiver. From this point of view only minimum gain, which suppresses the noise contribution of later stages, should be applied. Second, gain is needed so that the weakest input signal appearing at the ADC input is sufficiently greater than the quantization noise of the ADC. For DDSRs, however, the quantization noise profile is shaped by $\Delta \Sigma$ modulator (DSM) feedback. A properly designed DSM feedback ensures that the quantization noise in desired band is much lower than thermal noise floor, resulting in reduced dependency of the RX gain on ADC signal to noise ratio (SNR) requirements.

Based on the aforementioned discussion, our proposed DDSR consists of optimized receiver gain design together with tunable bandpass filtering at RF input. In coming sections we detail the design of RF front-end and DDSR loop filter.

\section{RECEIVER DESIGN}

\section{A. Front-end Design}

Figure 2 shows the block diagram of the proposed DDSR. Blocker filtering at the input is implemented by designing a low intrinsic input impedance $\left(\mathrm{Z}_{\mathrm{IN}}\right)$ Common-Gate CommonSource (CG-CS) LNA while for desired frequency $Z_{\mathrm{IN}}$ is boosted to match source impedance $\left(\mathrm{R}_{\mathrm{S}}\right)$ through implementation of up-converted positive feedback from baseband (BB). The amount of positive feedback can be adjusted through the resistance $R_{\text {POS. Neglecting any non idealities, the overall }}$ receiver input impedance of LNA can then be approximated as:

$$
Z_{I N}\left(f_{L O}\right)=\frac{Z_{L N A}}{1-\gamma A_{V}\left(\frac{Z_{I N}}{Z_{I N}+R_{P O S}}\right)}
$$

where $Z_{\mathrm{IN}}\left(f_{\mathrm{LO}}\right)$ is the input impedance at the desired frequency, $\mathrm{Z}_{\mathrm{LNA}}$ is the intrinsic input impedance of the LNA, $\mathrm{A}_{\mathrm{V}}$ denotes the voltage gain of the DDSR and $\gamma$ represents passive mixer conversion losses.

\section{B. DSM Design}

DSM filter design starts by dividing the gain in the RX chain. For optimized blocker rejection, minimum required gain is applied only in the first stage of DDSR loop filter. After this, the number of stages required for quantization noise shaping can be derived from [4]:

$$
n=\frac{\log \left(\frac{\Delta^{2}}{6 f_{s} k T G_{R X} F_{R X}}\right)}{2 \log \left(\frac{f_{N S P}}{f_{B W}}\right)}
$$

where $\Delta$ is the quantizer step size, $\mathrm{f}_{\mathrm{S}}$ is the sampling frequency, $\mathrm{K}$ is Boltzmann constant, $\mathrm{T}$ is the temperature in Kelvins, $G_{R X}$ is the RX power gain, $F_{R X}$ is the noise factor of the RX excluding quantization noise, $\mathrm{f}_{\mathrm{BW}}$ is $\mathrm{BB}$ bandwidth cutoff frequency, and $\mathrm{f}_{\mathrm{NSP}}$ is the combined noise shaping pole cutoff frequency. $\mathrm{f}_{\mathrm{NSP}}$ is selected such that the quantization noise is attenuated far below the thermal noise floor inside the desired band.

For an initial $\mathrm{f}_{\mathrm{NSP}}$ selection at 10 times $\mathrm{f}_{\mathrm{BW}}$, we can calculate required noise shaping poles as $n=2.57$ or 3 . Apart from noise shaping, channel select filtering (CSF) is also needed from DSM loop filter. As gain is only applied in a single stage, a single CSF pole is positioned at $\mathrm{f}_{\mathrm{BW}}$. This CSF pole and three noise shaping poles create a fourth order DSM loop filter which was implemented through a cascade-of-integrators in feedback (CIFB) topology, where the first stage provides channel select filtering and the other three stages provide noise shaping for the DDSR. As an initial value for the DSM filter, Butterworth filter coefficients were chosen due to minimal peaking in its signal transfer function (STF) response. Final filter coefficients values after scaling with stage gains and referring to $f_{B W}$ are shown in Figure 2(a). 
In the proposed DDSR, an additional positive feedback from the DDSR baseband is needed for input impedance matching. To keep the DSM filter design simple and to avoid the risk of instability, positive feedback was designed separately after the 4th order DSM implementation. Positive feedback increases the RX gain and hence reduces BB channel bandwidth. Therefore, initial design of the DSM loop filter starts with smaller gain and selection of $f_{B W} 1.5$ times than desired $f_{B W}=10 \mathrm{MHz}$ so that positive feedback reduces the bandwidth to the desired $\mathrm{f}_{\mathrm{BW}}$ after implementation.

In order to avoid risk of quantization noise up-conversion through positive feedback quadrature mixers, $\mathrm{f}_{\mathrm{S}}=\mathrm{f}_{\mathrm{LO}}$ was selected. This ensured that the up-converted quantization noise falls out of the desired band and causes minimal NF degradation. The quantizer/IDACs for DDSR were implemented as ideal 4bit components with $600 \mathrm{mV}$ differential input range. A clock delay of $200 \mathrm{ps}$, for $\mathrm{f}_{\mathrm{S}}=\mathrm{f}_{\mathrm{LO}}$, was added between the quantizer output and the IDACs input for modeling real clock skew.

\section{SySTEM IMPLEMENTATION}

The DDSR is designed for an RX gain of $20 \mathrm{~dB}$. The RF front-end of DDSR consists of an LNA implementation with a parallel combination of CG and push-pull CS amplifiers [5]. The parallel combination increases the LNA gm LNA $_{\text {, and push- }}$ pull configuration helps to achieve better large signal linearity. The differential intrinsic input impedance of the LNA was designed to be around $25 \Omega$ differential by increasing the single ended CG $g_{m}$ from usual $20 \mathrm{mS}$ to be $40 \mathrm{mS}$.

Positive feedback from the output of the BB amplifiers is up-converted to the RF nodes through passive quadrature mixers. The amount of positive feedback is controlled through series resistors $R_{P O S}$. The stability of positive feedback loop was analyzed through transient and simple pole-zero transfer function analysis [6]. Results confirmed stability for $R_{P O S}>$ $250 \Omega$. For design implementation, we selected $R_{P O S}=325 \Omega$, which ensured stability at all process corners.

Transistors with a large aspect-ratio of $48 / 0.03 \mu \mathrm{m}$ were implemented in the main path mixers. This ensured a small switch resistance of $8 \Omega$ for better attenuation at far away offsets from $f_{\mathrm{LO}}$. As there was no such requirement of smaller $\mathrm{R}_{\mathrm{ON}}$ for the positive feedback mixers, their aspect-ratio was selected to be four times smaller than main path mixers.

BB integrators were implemented through self-biased differential pair configurations. The first BB integrator is designed for a higher transconducatnce of $25 \mathrm{mS}$ for lower noise contribution. The later baseband stages are designed with a smaller transconductance of $3 \mathrm{mS}$. Capacitors $\mathrm{C}_{1}-\mathrm{C}_{4}$ implement the required $\mathrm{BB}$ bandwidth cutoff frequency $\left(\mathrm{f}_{\mathrm{BW}}\right)$ and noise shaping pole cutoff frequency $\left(\mathrm{f}_{\mathrm{NSP}}\right)$ in the DSM filter. Their values were selected based on the final tuned Butterworth coefficients.

\section{Performance Evaluation}

The proposed DDSR was evaluated in a $28 \mathrm{~nm}$ fully-depleted silicon-on-insulator (FD-SOI) process through transient and

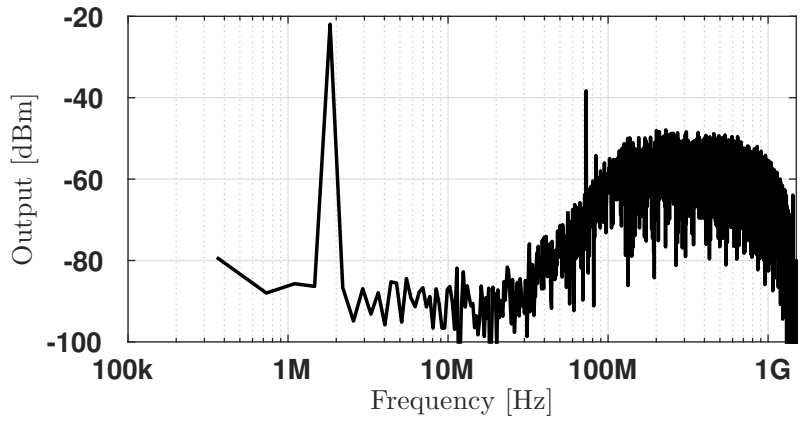

Fig. 3. Output spectrum at $\mathrm{f}_{\mathrm{LO}}=\mathrm{f}_{\mathrm{CLOCK}}=1.5 \mathrm{GHz}$, Signal power $\mathrm{P}_{\mathrm{IN}}=$ $-43 \mathrm{dBm} @ \mathrm{f}_{\mathrm{LO}}+1.83 \mathrm{MHz}$, Blocker power $\mathrm{P}_{\mathrm{BLOCKER}}=-43 \mathrm{dBm} @ \mathrm{f}_{\mathrm{LO}}+73 \mathrm{MHz}$ and $\mathrm{BB}$ bandwidth of $10 \mathrm{MHz}$.

steady state analysis. The RX is reconfigurable from 0.7 $2.7 \mathrm{GHz}$ with a BB bandwidth of $10 \mathrm{MHz}$. Further, based on our previous implementation [4], we added estimated values of PCB and layout parasitics, I/O pad capacitances, bondwire inductances and s-parameter models of $20 \mathrm{nH}$ off-chip RFchokes, and clock delay between quantizer and IDACs in order to match the simulated results more closely to the real scenario.

The spectrum of the the DDSR output bit stream is shown in Fig. 3. First, the desired in-band signal is amplified with around $20 \mathrm{~dB}$ of RX gain. Second, the blocker is attenuated by the channel select filtering (CSF) response and third, the quantization noise is shaped by the DDSR feedback loop such that there is little in-band quantization noise left.

Figures 4 show the RX SNDR versus input signal and blocker powers. the RX achieves a maximum SNDR of $53 \mathrm{~dB}$ at $\mathrm{P}_{\mathrm{IN}}$ of $-43 \mathrm{dBm}$. Further, a decrease in SNDR is observed at blocker input powers greater than $-35 \mathrm{dBm}$ for an input signal power of $-80 \mathrm{dBm}$. At low signal powers, the difference between ideal and simulated SNDRs is approximately equal to RX NF.

Figure 5 shows the simulated steady-state analysis results of DDSR gain and $S_{11}$. During the steady state simulations, the effects of quantizer and DACs were not considered. However, the results still reasonably match with the DDSR output spectrum in Figure 3. As desired, RX gain of $20 \mathrm{~dB}$ is observed within $20 \mathrm{MHz}$ RF bandwidth. Further, a differential input impedance of $25 \Omega$ can be seen, which creates the required blocker attenuation at LNA input.

Figures 6 shows the simulated blocker input compression point (BCP), NF and RX gain. The RX achieves an BCP of $-11.5 \mathrm{dBm}$ at $100 \mathrm{MHz}$ offset from the $\mathrm{f}_{\mathrm{LO}}$ with a maximum receiver integrated NF of $6.2 \mathrm{~dB}$.

\section{CONCLUSION}

A blocker resilient DDSR, with a low intrinsic input impedance front-end and optimized receiver gain, has been proposed which reduces the blocker gain at LNA input and output nodes. This ensures that the voltage swing limits are pushed towards much stronger blocker signals. For the desired frequency, input impedance is boosted to matched condition 
TABLE I

PERFORMANCE SUMMARY AND COMPARISON

\begin{tabular}{|c|c|c|c|c|c|}
\hline & 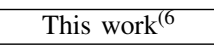 & [3] & [4] & [1] & [2] \\
\hline Carrier Frequency $(\mathrm{GHz})$ & $0.7-2.7$ & $0.6-3$ & $0.7-2.7$ & 0.9 & $0.1-1.5$ \\
\hline Gain $(\mathrm{dB})$ & 20 & 50 & - & 40 & 38 \\
\hline Noise Figure $(\mathrm{dB})$ & $5.6-6.2$ & $2.4-3.5$ & $5.9-8.8$ & 6.2 & $1.5-2.9$ \\
\hline Blocker input $\mathrm{P} 1 \mathrm{~dB}^{(1}(\mathrm{dBm})$ & $-11.5 @ 10 f_{\mathrm{BW}}$ & $-20 @ 10 f_{\mathrm{BW}}$ & $-14 @ 10 \mathrm{f}_{\mathrm{BW}}{ }^{(2}$ & $-18 @ 20 f_{\mathrm{BW}}$ & $-17 @ 10 f_{\mathrm{BW}}$ \\
\hline OB IIP3 $(\mathrm{dBm})$ & $0 @ 10 f_{\mathrm{BW}}$ & $-10 @ 10 f_{\mathrm{BW}}$ & $-4 @ 10 \mathrm{f}_{\mathrm{BW}}{ }^{(2}$ & - & - \\
\hline Peak SNDR $(\mathrm{dB})$ & 53 & 52 & 43 & 56 & - \\
\hline BB bandwidth ( $\left.\mathrm{f}_{\mathrm{BW}}\right)(\mathrm{MHz})$ & 10 & 10 & 7.5 & $28^{(3}$ & 2 \\
\hline Power (mW) @ Supply voltage (V) & $25 @ 1^{(5}$ & $35.5-53.5 @ 1.2$ & $90 @ 1.1$ & $80 @ 1.2$ & $11 @ 0.7,1.2^{(4}$ \\
\hline Architecture & LNA first DDSR & LNTA first DDSR & LNA first DDSR & LNA first DDSR & mixer first receiver \\
\hline Process & 28nm FDSOI & $65 \mathrm{~nm} \mathrm{CMOS}$ & 40nm CMOS & 65nm CMOS & 65nm CMOS \\
\hline
\end{tabular}

1) Blocker offset normalized to BB bandwidth ( $\left.f_{B W}\right)$ 2) Extrapolated value 3) Usable BW with acceptable NF is limited to $4 \mathrm{MHz} 4$ ) at RF 5) Power consumption of RX excluding quantizer and IDACs 6) Simulated response

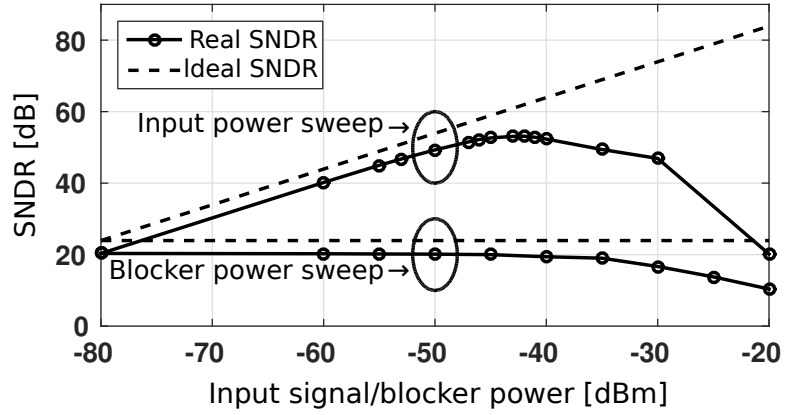

Fig. 4. SNDR vs. input and blocker power sweep for $\mathrm{f}_{\mathrm{LO}}=1.5 \mathrm{GHz}$

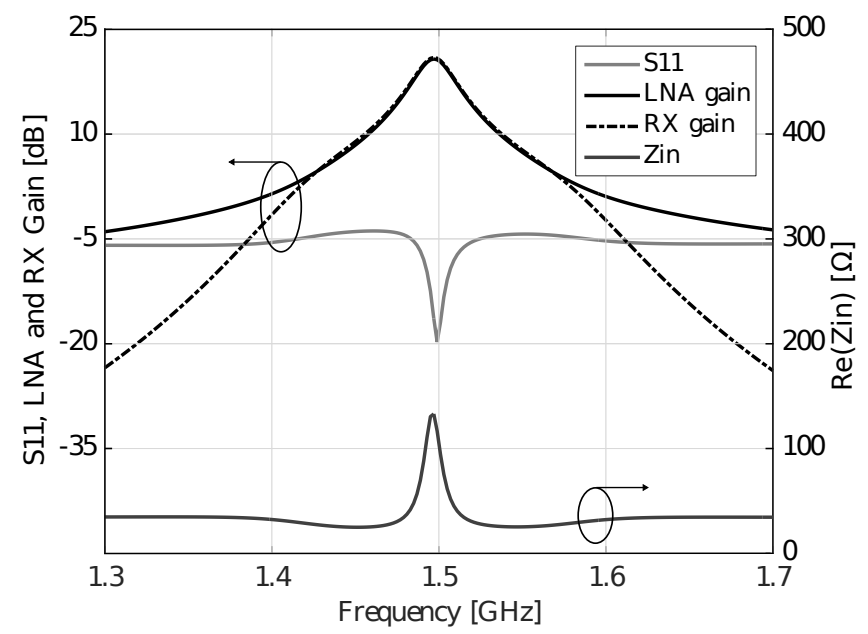

Fig. 5. Simulated $\mathrm{S}_{11}$, LNA and $\mathrm{BB}$ gain for $\mathrm{f}_{\mathrm{LO}}=1.5 \mathrm{GHz}$

through a positive up-converted feedback from BB. Simulated results show a OB-P1dB point of $-11.5 \mathrm{dBm}$ at $100 \mathrm{MHz}$ offset from the desired received frequency, with maximum SNDR of $53 \mathrm{~dB}$ and maximum NF of $6.2 \mathrm{~dB}$. Results collected into Table I indicate the proposed approach is able to achieve state-of-the art blocker tolerance even with lower supply voltage.

\section{ACKNOWLEDGMENT}

This research has received funding from the Academy of Finland.
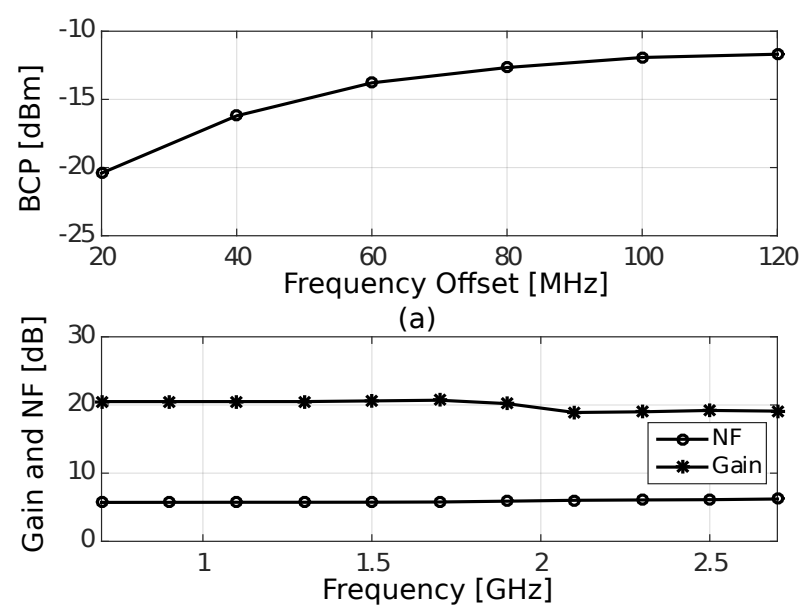

(b)

Fig. 6. (a) Blocker compression point (BCP) vs. frequency offset for the proposed receiver at $\mathrm{f}_{\mathrm{LO}}=1.5 \mathrm{GHz}$. (b) $\mathrm{RX}$ gain and noise figure across the $0.7-2.7 \mathrm{GHz}$ operating band.

\section{REFERENCES}

[1] K. Koli, J. Jussila, P. Sivonen, S. Kallioinen, and A. Pärssinen, "A $900 \mathrm{MHz}$ direct delta-sigma receiver in 65nm CMOS," in 2010 IEEE International Solid-State Circuits Conference - (ISSCC), Feb 2010, pp. 64-65.

[2] Z. Lin, P. I. Mak, and R. P. Martins, "A $0.028 \mathrm{~mm} 211 \mathrm{~mW}$ single-mixing blocker-tolerant receiver with double-RF N-path filtering, S11 centering, $+13 \mathrm{dBm}$ OB-IIP3 and 1.5-to-2.9dB NF," in 2015 IEEE International Solid-State Circuits Conference - (ISSCC) Digest of Technical Papers, Feb 2015, pp. 1-3.

[3] A. Nejdel, X. Liu, M. Palm, L. Sundström, M. Törmänen, H. Sjöland, and P. Andreani, "A $0.6-3.0 \mathrm{GHz} 65 \mathrm{~nm}$ CMOS radio receiver with deltasigma based A/D-converting channel-select filters," in European SolidState Circuits Conference (ESSCIRC), ESSCIRC 2015 - 41st, Sept 2015, pp. 299-302.

[4] M. Englund, K. Östman, O. Viitala, M. Kaltiokallio, K. Stadius, K. Koli, and J. Ryynänen, "A programmable $0.7-2.7 \mathrm{GHz}$ direct $\Delta \Sigma$ receiver in $40 \mathrm{~nm}$ CMOS," IEEE Journal of Solid-State Circuits, vol. 50, no. 3, pp. 644-655, March 2015.

[5] Z. Ru, N. A. Moseley, E. A. M. Klumperink, and B. Nauta, "Digitally enhanced software-defined radio receiver robust to out-of-band interference," IEEE Journal of Solid-State Circuits, vol. 44, no. 12, pp. 33593375, Dec 2009.

[6] K. Östman, M. Englund, O. Viitala, K. Stadius, K. Koli, and J. Ryynänen, "Characteristics of LNA operation in direct delta-sigma receivers," IEEE Transactions on Circuits and Systems II: Express Briefs, vol. 61, no. 2, pp. 70-74, Feb 2014. 